\title{
Experimental Study of Resonance Frequency at Prime Mover Thermoacoustic Standing Wave
}

\author{
Danang D. Cahyadi ${ }^{1}$, Yoga N. Adhitama, Ikhsan Setiawan, Agung B. S. Utomo \\ Departement of Physics University of Gadjah Mada \\ Sekip Utara BLS 21 Yogyakarta 55281 Indonesia \\ 1E-mail: danang.dwi.c@mail.ugm.ac.id
}

Received 29 August 2017, Revised 29 September 2017, Accepted 2 October 2017

\begin{abstract}
Thermoacoustic prime movers work by using thermal energy to produce acoustic energy in the form of sound wave through thermoacoustic effect which occurs in a porous medium called stack. This paper describes an experimental study on the relation between the order of resonance frequencies generated by a thermoacoustic prime mover and the length of the resonator and the viscous penetration depth. Extending the resonator length will decreasing the resonance frequency which result in the increasing in the viscous penetration depth. Generally, the generated sound consists of only one frequency, that is the first-order one. However, under certain conditions, the sound has only the second-order frequency or comprises two frequencies of the first-order and second-order resonance frequencies. This phenomenon can be explained by considering the comparison between the effective hydraulic radius of stack $\left(r_{\mathrm{ef}}\right)$ and the viscous penetration depth $\left(\delta_{\mathrm{v}}\right)$. It is found that the first-order frequency appears when $\mathrm{r}_{\mathrm{ef}}>\delta_{\mathrm{v}}$, while when $\mathrm{r}_{\mathrm{h}}<\delta_{\mathrm{v}}$ (with $\delta_{\mathrm{v}}$ calculated by using the first-order frequency) then the second order frequency is produced so that $\delta_{\mathrm{v}}$ is back to a smaller value and therefore the condition of $\mathrm{r}_{\mathrm{h}}>\delta_{\mathrm{v}}$ is recovered. In addition, when of $\mathrm{r}_{\mathrm{ef}} \approx \delta_{\mathrm{v}}$ the thermoacoustic prime mover will generate the first and second order frequencies together.
\end{abstract}

Keyword: prime mover thermoacoustic, standing wave, resonance frequency, effective hydraulic radius, viscous depth penetration

\section{Introduction}

Thermoacoustics is a field of study of interaction between heat and sound, that is about conversion of thermal energy into acoustic energy and vice versa. The device that converts thermal energy into acoustic energy is called thermoacoustic prime mover, while that pumps heat from a low temperature reservoir to a high temperature one is called thermoacoustic heat pump or refrigerator.

Thermoacoustic devices have attracted the interest and attention of scientists in recent decades because they are environmentally friendly, have simple structure and high reliability, and can be made and maintained easily (Setiawan, 2016). The operation of thermoacoustic prime movers can use sunlight (Chen \& Garret, 1998; Adeff \& Hoffler, 2000) or waste heat (Gardner \& Howard, 2009) as the source of thermal energy, and does not produce any exhaust gases such as carbon dioxide as in 
conventional machines. On the other hand, thermoacoustic refrigerators typically use inert gases like air and noble gases as their working fluid (Tijani, 2001) instead of freon as in conventional refrigerators.

Thermoacoustic prime movers generally have a typical deficiency, that is low efficiency. So far, the maximum thermal efficiency of termoacoustic prime mover of traveling wave type is $30 \%$ but with more complex structure and equipment such as a jet pump (Backhauss \& Swift, 2000). In addition, standing wave type thermoacoustic prime movers have so far $20 \%$ thermal efficiency (Swift, 1992; Backhauss \& Swift; 2000) because they works with a thermodynamic cycle that is intrinsically irreversible. The traveling wave thermoacoustic prime movers have a higher efficiency because the operates with Stirling cycle which is inherently reversible.

Although thermoacoustic prime movers have low thermal efficiency, the ability of these devices to work by using waste heat makes the application of thermoacoustic prime movers would be attractive and useful to improve the overall thermal system (Setiawan, 2015). Thermoacoustic prime movers are usually applied to generate electrical energy by combining it with a linear alternator (Backhauss et al, 2004; Kitadani et al, 2010). On the other hand, when a thermoacoustic prime mover is combined with a thermoacoustic stack (or regenerator), it forms a thermoacoustic cooling system (refrigerator) without moving parts (Yu et al, 2011; Saechan et al., 2013).

In its application, the sound produced by the thermoacoustic prime mover have an important role as the energy source for generating electricity and driving a thermoacoustic refrigerator. One of the important parameters of the generated sound wave is the resonant frequency. This paper describes an experimental study about the change of resonant frequency generated by a standing wave thermoacoustic prime mover due to the variation of the resonator length. It is found that with a certain resonator length only the first order frequency is generated, while with other resonator lengths, the first- and second- order frequency are produced together or only the second order appears. This paper explains these facts by relating to the effect of viscous penetration depth of the working gas in determining the output resonant frequency.

\section{The Thermoacoustic Principle}

The thermoacoustic prime mover mainly consists of a resonator tube, a stack that is a porous medium, a working gas and two heat exchangers. Resonator tubes are filled with working gases such as air, noble gases or other inert gases. The stack is flanked by a hot heat exchanger (HHX) and an ambient heat exchanger (AHX). This combination of stack and two heat exchangers are placed inside the resonator tube. Both heat exchangers will provide a large temperature gradient along the stack in the direction of the resonator axis. The temperature gradient is required for thermoacoustic energy conversion processes to occur. The minimum temperature difference between the two ends of the stack required to initiate spontaneous oscillation of working gas (producing sound wave) is called the onset temperature difference $\left(\Delta T_{\text {onset }}\right)$. 
The process of the sound waves generation can be explained by following the things that happen to a gas parcel inside a stack's pore as shown in Figure 1. It is assume that the distance of the gas parcel and the wall of the pore is in order to the thermal penetration depth $\left(\delta_{\kappa}\right)$ of theworking gas. In this case, imperfect thermal contact between the gas parcel and the wall occurs. Therefore, the gas parcel is undergoing a thermodynamic cycle consisting of four stages (Setiawan, 2015 ). In step (1) the gas parcel is heated due to absorbing a certain amount of heat $d Q_{1}$ and undergoing thermal expansion. In this case, work $d W_{1}$ is done by gas parcell. In step (2), due to the expansion process, this gas parcel spreads to the cold side and does work of $d W$. This process causes the hot side area run into pressure drop. In step (3), Upon reaching the cold side, as the gas parcel is hotter than the wall of the stack pores, the gas parcel releases the heat $d Q_{2}$ to the stack and undergoes cooling and thermal contraction. In this case, the $d W_{2}$ is the work done on the gas parcel. In step (4), the gas parcel returns to the hot side to fill the low pressure area and undergoes compression, and the work $d W$ is done on the gas parcel. The gas parcel will repeat the cycle starting from the thermal expansion process in step (1).

(1)

(2)
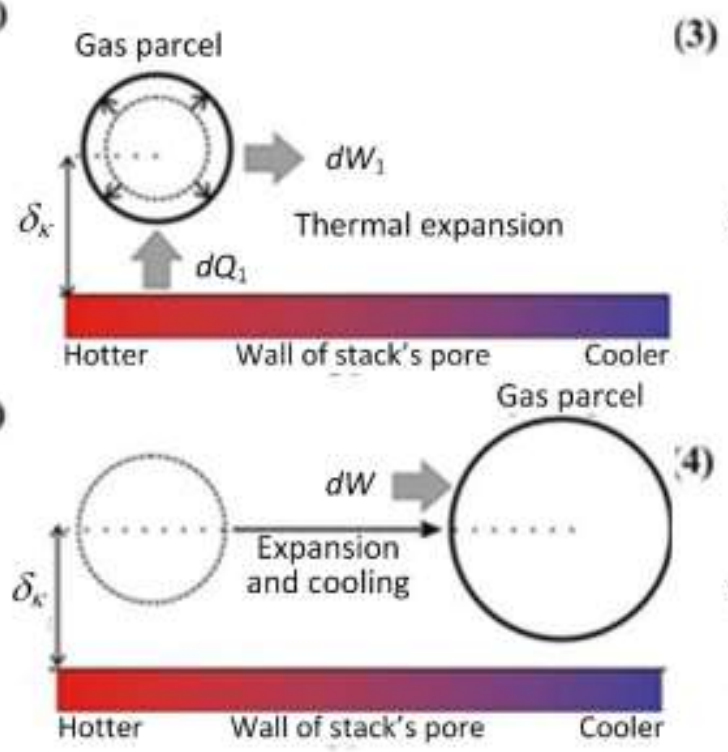

(3)
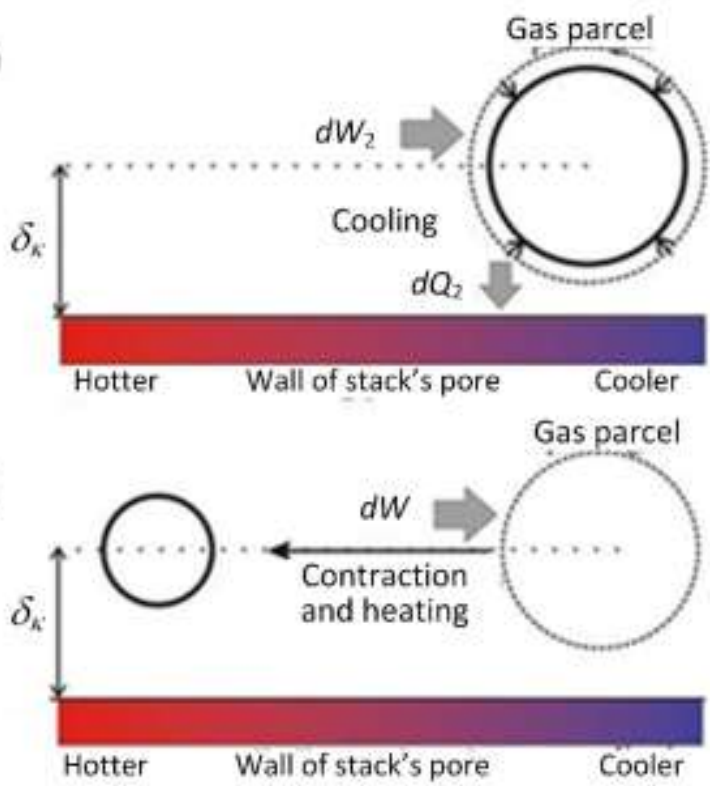

Figure 1. Four stages of thermoadynamic process experienced by the gas parcel.

Figure 1. shows the schematic of pressure-volume diagram for the four thermodynamic processes undertaken by the gas parcel as described above (Fig. 1), known as the Brayton cycle (Setiawan, 2015). The resulting closed-loop area $\oint p d V$ indicates the total work used by the standing-wave thermoacoustic prime mover to produce acoustic power (In't Panhuis, 2009). 


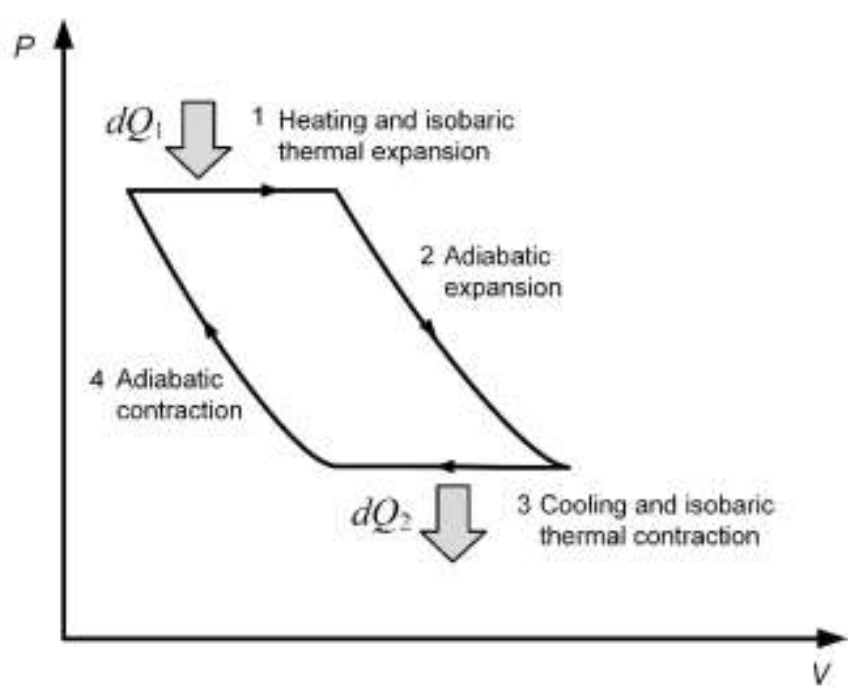

Figure 2. The schematic pressure-volume $(P-V)$ diagram of the thermodynamic cycle executed by the gas parcel.

The resonator used in the study is a straight tube made of stainless-steel pipe with one end open. The $n$-th order resonant frequency is

$$
f_{n}=(2 n-1) \frac{v}{4 L} \text {. }
$$

where $n=1,2,3, \ldots, L$ is the resonator length, $v$ is the sound speed in air.

The viscous penetration depth is the distance of the viscosity frictional force spreads over the time interval of one period of sound wave oscillation. The viscous penetration depth is measured from the wall the stack's pore and expressed by (Saechan, 2014)

$$
\delta_{v}=\sqrt{\frac{2 \mu}{\omega \rho_{m}}}=\sqrt{\frac{2 v}{\omega}}
$$

where $\mu$ and $v$ are dynamic viscosity $\left(1.8 \times 10^{-5} \mathrm{~N} \cdot \mathrm{s} \cdot \mathrm{m}^{-2}\right)$ and kinetic viscosities $(1.6 \times$ $\left.10^{-5} \mathrm{~m}^{2} \cdot \mathrm{s}^{-1}\right)$, respectively, $\omega$ is the angular frequency, and $\rho_{m}$ is the mean density of the working gas (atmospheric air) that is $1.17 \mathrm{~kg} \cdot \mathrm{m}^{-3}$. The effect of the viscosity of the working gas is attenuating the acoustic power.

In the conversion of thermal energy into acoustic energy, the required condition is that the effective hydraulic radius $\left(r_{\mathrm{ef}}\right)$ of the stack's pore is greater than the viscous penetration depth $\left(r_{\mathrm{ef}}>\delta_{v}\right)$, so that the negative effect of the viscosity will be lower. In order to have $\delta_{v}$ small, the frequency ( $\omega$ or $f_{n}$ ) should be high. For a given sound speed $(v)$, a higher frequency can be achieved by using a longer resonator tube.

\section{Experimental Method}

The schematic diagram of the standing wave thermoacoustic prime mover with a straight resonator in this study is shown in Fig. 3. The resonator length is varied, those are $30 \mathrm{~cm}, 105 \mathrm{~cm}, 130 \mathrm{~cm}, 180 \mathrm{~cm}$, and $205 \mathrm{~cm}$. A stack, which is made of a pile of stainless-steel wire-mesh, is placed inside the resonator near the closed end. The calculated effective radius $\left(r_{\mathrm{ef}}\right)$ of the stack's pore is $0.275 \mathrm{~mm}$. The stack length is 30 
$\mathrm{mm}$. The stack is tightly sandwiched between a hot exchanger heat (HHX) and an ambient heat exchanger (AHX). Air at atmospheric pressure (1 atm) and room temperature $\left(27^{\circ} \mathrm{C}\right)$ is used as the working gas inside the resonator.

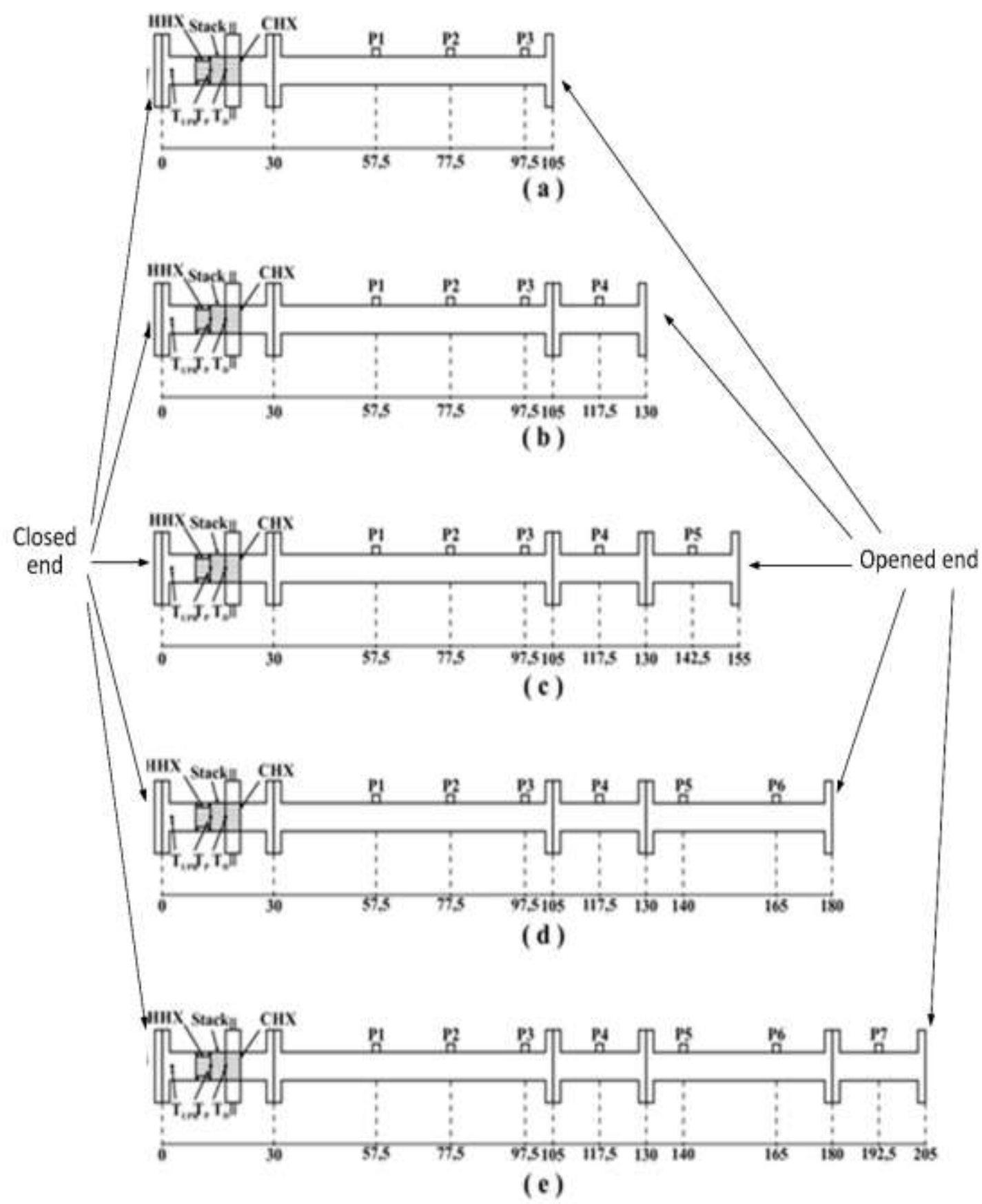

Figure 3. The schematic diagram of the standing-wave thermoacoustic prime mover with various resonator lengths and one of resonator end is open.

The sound produced by the prime mover thermoacoustic is generated by a large temperature gradient along the stack. The high temperature at the hot end of stack comes from HHX which is wound by an electric heating element (flexible cable heater) 
with 350 watt input electric power. While the lower temperature at the ambient end of stack is maintained by ambient water flowing through the AHX. The temperature different between the both ends of stack exictes the gas oscillation (sound wave) due to the thermoacoustic effect. The temperatures are measured by using K-type thermocouples which is connected to a data logger and computer.

Pressure transducers are mounted at the resonator wall to measure the dynamic pressure of the sound inside the resonator at different location as shown in Fig. 3 indicated as P1 until P7. The sound pressure data is monitored and recorded by using a data logger and computer. The data is analyzed by using FFT (Fast Fourier Transform) to obtain the frequency spectrum of the generated sound.

\section{Results and Discussion}

The frequency data of the sound generated by the thermoacoustic prime mover with various resonator lengths are shown in Fig. 4. It can be seen that there are two groups of frequency. The first is corresponding to Eq. (1) with $n=1$ (the first order resonance), i.e. $87 \mathrm{~Hz}$ and $68 \mathrm{~Hz}$ which are given by the resonator lengths of $105 \mathrm{~cm}$ and $130 \mathrm{~cm}$, respectively. The second is corresponding to Eq. (1) with $n=2$ (the second order resonance), those are $199 \mathrm{~Hz}, 170 \mathrm{~Hz}, 147 \mathrm{~Hz}$, and $128 \mathrm{~Hz}$ which are provided by the resonator lengths of $130 \mathrm{~cm}, 155 \mathrm{~cm}, 180 \mathrm{~cm}$, and $205 \mathrm{~cm}$, respectively. It is interesting to find that the resonator with $130 \mathrm{~cm}$ long produces sound with two frequencies and the longer resonator selects the second order resonance $(n=2)$ instead of the first one $(n=$ 1). These results can be explained by considering the effect of the viscosity of gas within the stack's pores, that is the ratio of the viscous penetration depth $\left(\delta_{v}\right)$ of the working gas to the efective radius $\left(r_{\mathrm{ef}}\right)$ of the pores, as described below.

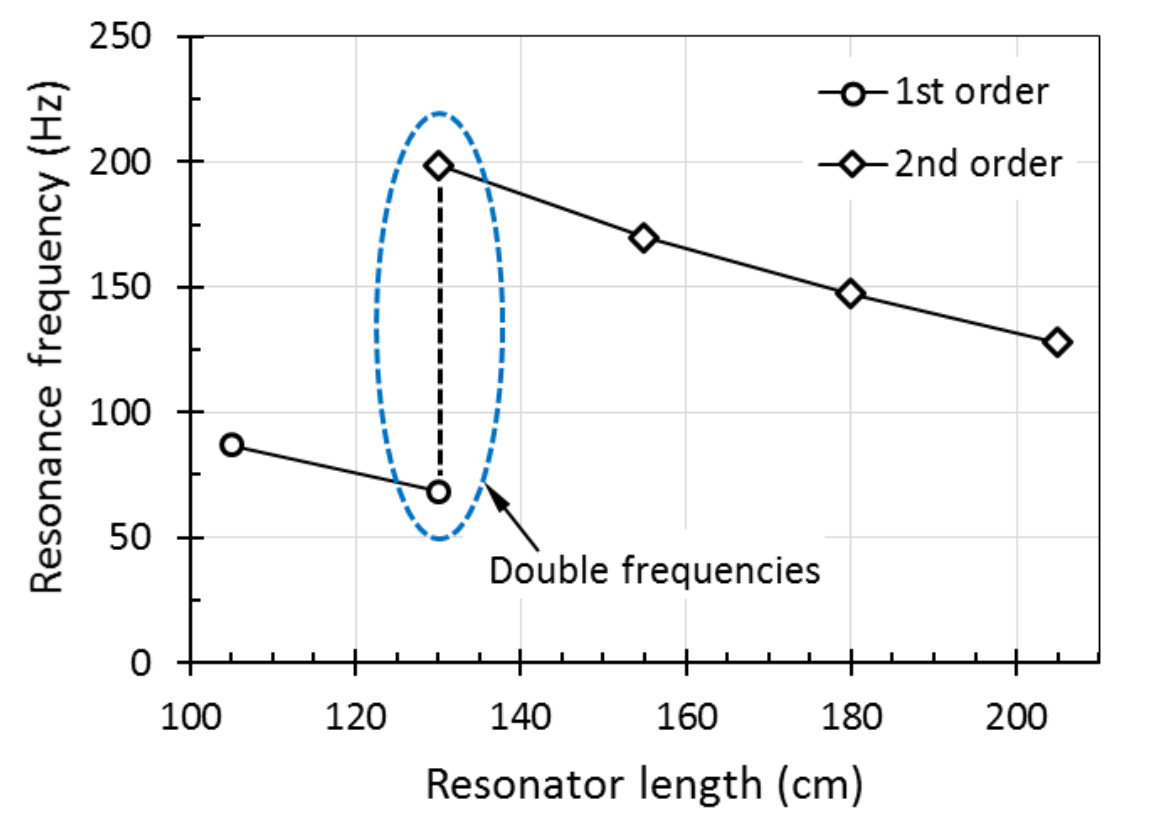

Figure 4. Relationship between resonator length and resonance frequency. 


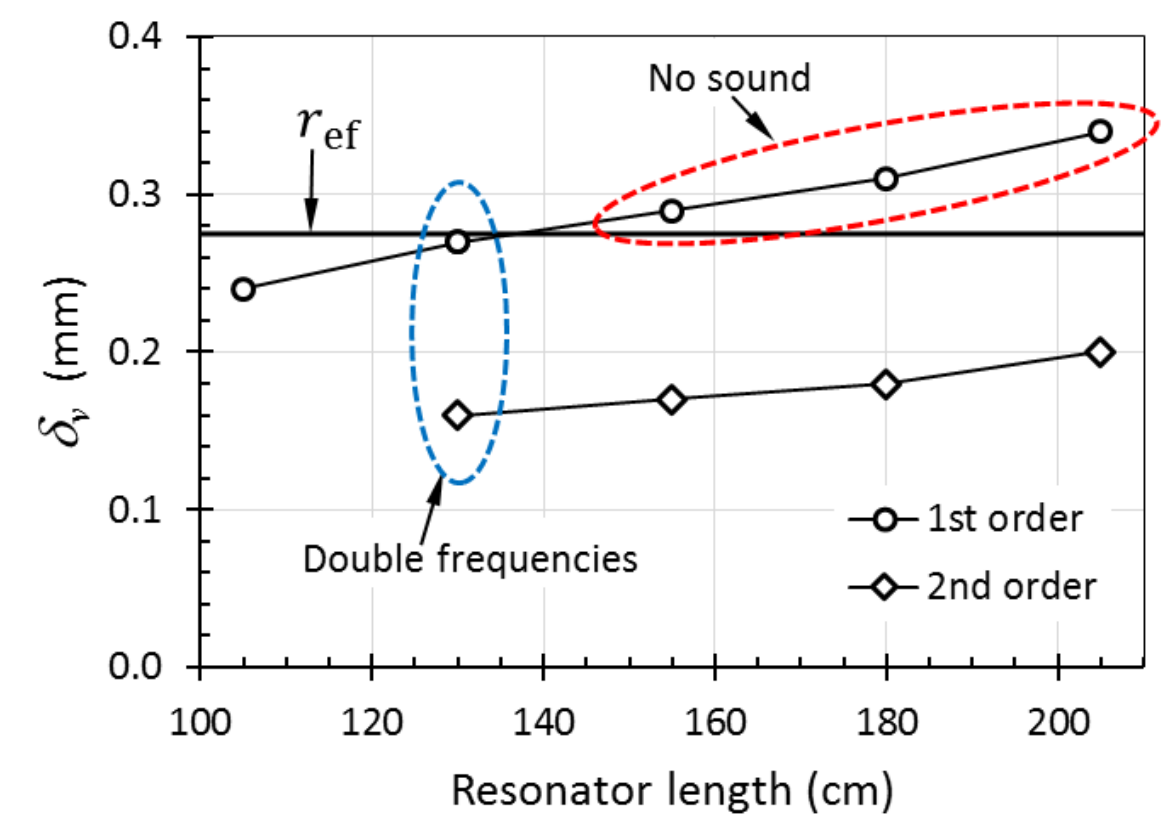

Figure 5. Relationship between resonator length and viscous penetration depth parameter $\left(\delta_{v}\right)$.

Figure 5 shows the values of viscous penetration depth $\left(\delta_{v}\right)$ which are calculated by using Eq. (2) and the first- and second order resonance frequencies of the resonator with various lengths mentioned above. The first order frequency of the $105 \mathrm{~cm}$ resonator is $87 \mathrm{~Hz}$, giving $\delta_{v}=0.24 \mathrm{~mm}$. Because $r_{\mathrm{ef}}=0.275 \mathrm{~mm}$, then in this case $\delta_{v}<r_{\mathrm{ef}}$, the viscous effect is relatively small and therefore the thermoacoustic prime mover is able to produce the sound with frequency of $87 \mathrm{~Hz}$.

On the other hand, the first order frequency of the resonator with length of $155 \mathrm{~cm}$, $180 \mathrm{~cm}$, and $205 \mathrm{~cm}$ is calculated as $56 \mathrm{~Hz}, 49 \mathrm{~Hz}$, and $43 \mathrm{~Hz}$, resulting in $\delta_{v}$ of 0.29 $\mathrm{mm}, 0.31 \mathrm{~mm}$, and $0.34 \mathrm{~mm}$, respectively. Thus, in this case, $\delta_{v}>r_{\mathrm{ef}}$, the viscous effect is relatively large and therefore the thermoacoustic prime mover is not able to produce the sound with those low frequencies. To overcome this problem, the prime mover then select a higher resonance frequency, that is the second order frequency ( $n=$ 2 ), to have $\delta_{v}<r_{\text {ef. }}$. The second order frequencies which are found in this experiment for the corresponding resonator length are $170 \mathrm{~Hz}, 147 \mathrm{~Hz}$, and $128 \mathrm{~Hz}$, giving $\delta_{v}$ of $0.17 \mathrm{~mm}, 0.18 \mathrm{~mm}$, and $0.20 \mathrm{~mm}$, respectively. We can see now in this case that $\delta_{v}<r_{\text {ef }}$ which means the viscous effect is relatively small and therefore the thermoacoustic prime mover is able to produce the sound with the second order requencies. This result is similar to that of Sakamoto and Watanabe (2006) that the second order frequency is generated by thermoacoustic prime mover because the working gases adjust from the viscous penetration depth by increasing their frequency to produce sound. The difference is that their experiment was carried out with a traveling wave thermoacoustic device with looped-tube configuration. 
A special result is found in this experiment with resonator length of $130 \mathrm{~cm}$. In this case, the prime mover produces sound that is consisting of two different frequencies, those are $68 \mathrm{~Hz}\left(1^{\text {st }}\right.$ order $)$ and $199 \mathrm{~Hz}\left(2^{\text {nd }}\right.$ order $)$. This fact is understood as because the value of viscous penetration depth of gas calculted using the first order frequency is almost the same as the effective radius of the stack's pore, that is $\left(\delta_{v} \approx r_{\mathrm{ef}}\right)$. In the other word, this is a transitional condition for the prime mover between producing the first order and producing the second order of sound frequencies.

In addition to the influence of resonator length on the frequency of the generated sound, we also examine the effect of resonator length on the pressure amplitude of the sound wave, as depicted in Fig. 6. It can be seen that the pressure amplitude tends to be higher (from 1,97 $\mathrm{kPa}$ up to $3.33 \mathrm{kPa}$ ) when the resonator length gets longer (from 105 $\mathrm{cm}$ until $205 \mathrm{~cm}$ ), except at the transitional condition (resonator length of $130 \mathrm{~cm}$ ). At the transitional condition, which has two components of frequency, the both components have pressure amplitudes lower than those of other sound with only one frequency component. This is occurred because the sound energy is shared between the both components of different frequencies.

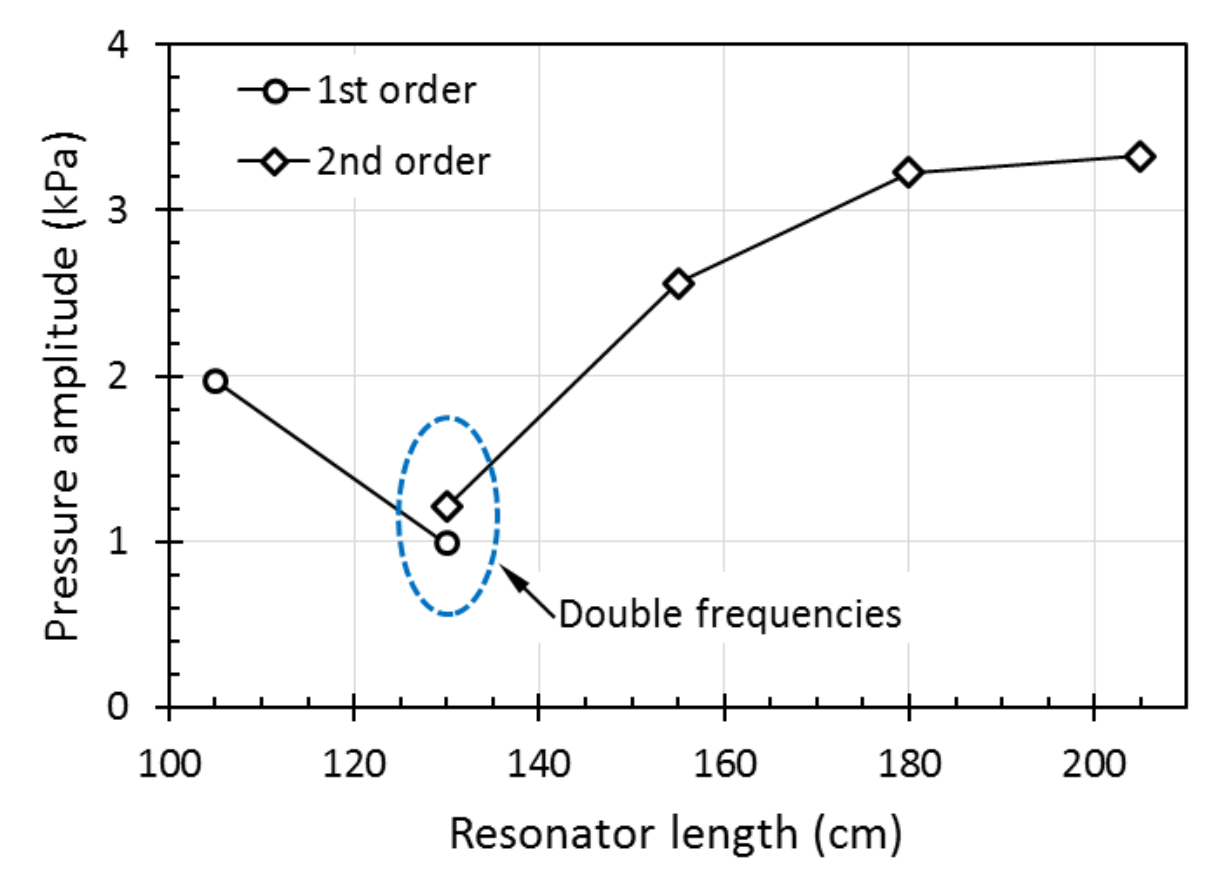

Figure 6. Pressure amplitudes for various resonator lengths.

\section{Conclusion}

The order of the resonance frequency of the sound generated by the standing-wave thermoacoustic prime mover is determined by the ratio of the effective radius $\left(r_{\mathrm{ef}}\right)$ of the stack's pores to the viscous penetration depth $\left(\delta_{v}\right)$ of the working gas. The first order resonance frequency is produced when the effective radius is larger than the viscous penetration depth $\left(r_{\mathrm{ef}} / \delta_{v}>1\right)$. When the effective radius is almost the same as 
the viscous penetration depth $\left(r_{\mathrm{ef}} / \delta_{v} \approx 1\right)$, then the prime mover generates the sound which is consisting of two components of resonance frequency, those are the first and second order resonance frequencies. On the other hand, if the first order frequency will result in a large $\delta_{v}$ so that $r_{\mathrm{ef}} / \delta_{v}<1$, then the prime mover produce the sound with a higher order resonance frequency (e.g. the second order) in order to have a smaller $\delta_{v}$ and the condition of $r_{\mathrm{ef}} / \delta_{v}>1$ recovered.

\section{Reference}

Adeff, J. A., dan Hoffler T. J., 2000, Design and Construction of Solar-powered, Thermoacoustically Driven, Thermoacoustic Refrigerator, J. Acoust. Soc. Am. 107, L37-L42.

Backhaus, S., dan Swift G. W., 2000, A Thermoacoustic Stirling Heat Engine: Detailed Study, J. Acoust. Soc. Am. 107, 3148-3166.

Backhaus, S., Tward E., dan Petach, M., 2004, Traveling-wave Thermoacoustic Electric Generator, App. Phys. Lett. 85, 1085-1087.

Chen, R. L. dan Garret, S. L., 1998, Solar/Heat-driven Thermoacoustic Engine, J. Acoust. Soc. Am. 103, 2841.

Gardner, D. L., dan Howard C. Q., 2009, Waste-heat-driven Thermoacoustic Engine and Refrigerator, Proc. of Acoustics, Australian Acoust. Soc., Adelaide, Australia.

In't Panhuis, P. H. M. W., 2009, Mathematical Aspect of Thermoacoustics, Thesis, Universitas Teknik Eindhoven, Jerman.

Kitadani, Y., Sakamoto, S., Sahashi K., dan Watanabe, Y., 2010, Basic Studi for Practical Use of Thermoacoustic Electric Generation System, Proc. 20th Int'l Congr. Acoust., Australian Acoust. Soc., Sidney.

Saechan, P., Kang, H., Mao, X., dan Jaworski, A. J., 2013, Thermoacoustic Refrigerator Driven By A Combustion Powered Thermoacoustic Engine - Demonstrator Of Device For Rural Areas Of Develoving Countries, Proc. World Congr. Engineering, London, UK.

Sakamoto, S., dan Watanabe, Y., 2006, Experimental Study on Resonance Frequency of Loop-tube Type Thermoacoustic Cooling System, Acoust. Sci. \& Tech. 27, 6.

Setiawan, I., Murti, P., Achmadin W. N., Agung B. S. Utomo, and Nohtomi, M., 2015, Design, Construction and Evaluation of a Standing Wave Thermoacoustic Prime Mover, AIP Conf. Proc. 1717, 05007-1-050007.

Setiawan, I., Murti, P., Utomo, B. S., Achmadin, W. N., and Nohtomi, M., 2016, Experimental Study on a Standing Wave Thermoacoustic Prime Mover with Air as Working Gas at Various Pressures, J. Phys.: Conf. Ser. 710012031

Swift, G.W., Analysis and performance of a large thermoacoustic engine, J. Acoust. Soc. Am. 92, 1992, 1551-1563

Tijani, M. E. H., 2001, Loudspeaker-driven Thermoacoustic Refrigeration, Ph.D. dissertation, Technische Universiteit Eindhoven, Belanda. 
Yu, B., Luo, E. C., Li, S. F., Dai, W., dan Wu, Z. H., 2011, Experimental Study of A Thermoacoustically Driven Traveling Wave Thermoacoustic Refrigerator, Cryogenics 51, 49-54. 